\title{
Constraining sterile neutrino interpretations of the LSND and MiniBooNE anomalies with coherent neutrino scattering experiments
}

\author{
Carlos Blanco $\odot,{ }^{1,2}$ Dan Hooper, ${ }^{2,3,4}$ and Pedro Machado ${ }^{5}$ \\ ${ }^{1}$ Department of Physics, University of Chicago, Chicago, Illinois 60637, USA \\ ${ }^{2}$ Kavli Institute for Cosmological Physics, University of Chicago, Chicago, Illinois 60637, USA \\ ${ }^{3}$ Theoretical Astrophysics Group, Fermi National Accelerator Laboratory, Batavia, Illinois 60510, USA \\ ${ }^{4}$ Department of Astronomy and Astrophysics, University of Chicago, Chicago, Illinois 60637, USA \\ ${ }^{5}$ Theoretical Physics Department, Fermi National Accelerator Laboratory, Batavia, Illinois 60510, USA
}

(Received 14 February 2019; accepted 7 April 2020; published 29 April 2020)

\begin{abstract}
We propose to test the light sterile neutrino solution to the Liquid Scintillator Neutrino Detector (LSND) and MiniBooNE anomalies by measuring the coherent elastic scattering rate of neutrinos from a pulsed spallation source. Coherent scattering is universal across all active neutrino flavors, and thus can provide a measurement of the total Standard Model neutrino flux. By performing measurements over different baselines and making use of timing information, it is possible to significantly reduce the systematic uncertainties and to independently measure the fluxes of neutrinos that originate as $\nu_{\mu}$ or as either $\nu_{e}$ or $\bar{\nu}_{\mu}$. We find that a $100 \mathrm{~kg} \mathrm{CsI}$ detector would be sensitive to a large fraction of the sterile neutrino parameter space that could potentially account for the LSND and MiniBooNE anomalies.
\end{abstract}

DOI: $10.1103 /$ PhysRevD.101.075051

\section{INTRODUCTION}

A large number of neutrino oscillation experiments carried out over the past two decades have lead to the establishment of a standard framework, in which the neutrino sector is comprised of three massive neutrinos with relatively large mixing angles and subelectronvolt mass splittings. Some of the data that has been collected, however, cannot be explained within the context of this standard framework. Particularly puzzling have been the results of the Liquid Scintillator Neutrino Detector (LSND) [1] and MiniBooNE [2] experiments. In the former, an excess of $\bar{\nu}_{e}$ events was observed from a source of pions decaying at rest, while in the latter an excess of electronlike events was observed in both $\nu_{\mu}$ and $\bar{\nu}_{\mu}$ beams. Although these experiments bear very distinct neutrino energy profiles, their results are each consistent with $\nu_{\mu} \rightarrow \nu_{e}$ oscillations occurring over a short baseline, requiring a relatively large mass splitting [3-6]. Moreover, reexaminations [5,7-9] of the Gallium calibration experiments $[10,11]$ and reactor antineutrino fluxes [12,13] have identified a deficit of $\nu_{e}$ events relative to theoretical expectations.

Published by the American Physical Society under the terms of the Creative Commons Attribution 4.0 International license. Further distribution of this work must maintain attribution to the author(s) and the published article's title, journal citation, and DOI. Funded by SCOAP ${ }^{3}$.
Together, these anomalies appear to point to the existence of a sterile neutrino with a mass near the electronvolt scale. Complicating this interpretation, however, are the results of several experiments which measure $\nu_{\mu} \rightarrow \nu_{\mu}$ transitions. In particular, the IceCube [14] and MINOS/ MINOS+ [15] experiments should be sensitive to the presence of such a sterile neutrino, but have observed no deviation from the standard (three-neutrino) framework. Detailed statistical analyses of the combined short baseline neutrino data have revealed a large degree of tension, at the $4.7 \sigma$ level, between the appearance $\left(\nu_{\mu} \rightarrow \nu_{e}\right)$ and disappearance $\left(\nu_{\mu} \rightarrow \nu_{\mu}\right.$ and $\left.\nu_{e} \rightarrow \nu_{e}\right)$ datasets, at least within the context of oscillations with one or more sterile neutrinos $[3,16,17]$.

Motivated by this tension, a number of alternative explanations for the short baseline anomalies have recently been put forward. For example, models in which neutrinos upscatter to heavier fermions can provide a good fit to MiniBooNE's energy spectrum and angular distribution [18-21], but cannot account for the LSND excess. Other explanations involving "dark" particles produced in the beam have also been considered, but are strongly constrained by the MiniBooNE beam dump run data [22,23]. Moreover, scenarios that connect the neutrino mass generation to extra dimensions, leading to an infinite KaluzaKlein tower of sterile neutrinos, also seem to be disfavored by data [24-32]. Importantly, an alternative analysis of the LSND data finds that the potential signal may be at the edge of the detector's sensitivity [33]. At this time, the correct interpretation of this data remains unclear, and further 
information will be required in order to clarify this confusing situation.

In this paper, we propose to measure or constrain the occurrence of short-baseline active-to-sterile neutrino oscillations using neutrinos from a source of pions decaying at rest, which are then measured via neutral current coherent elastic neutrino-nucleus scattering. This interaction, which has recently been observed for the first time by the COHERENT Collaboration [34], using a CsI target [35], is universal across all three active neutrino flavors, and thus can provide a measurement of the total (all flavor) active neutrino flux [36,37] (see also Refs. [38-40]). Furthermore, by taking data on two different baselines, we can substantially reduce the systematic uncertainties associated with the detector efficiency, as well as with the overall flux and cross section $[38,41-43]$. The use of timing information provides information pertaining to neutrino flavor, allowing us to disentangle the effects of sterile neutrino mixing with $\nu_{\mu}$ and $\nu_{e}$, and thus providing a powerful probe of the LSND and MiniBooNE anomalies.

\section{COHERENT NEUTRINO SCATTERING}

For our experimental setup, we have in mind a source of neutrinos that is functionally similar to the Spallation Neutron Source at the Oak Ridge National Laboratory [44,45]. In particular, we consider a pulsed beam of $1 \mathrm{GeV}$ protons with a luminosity of $L_{0}=4 \times 10^{23} \mathrm{yr}^{-1}$, of which approximately $8 \%(6 \%)$ produce a $\pi^{+}\left(\pi^{-}\right)$upon striking the spallation target.

The high- $Z$ nature of the liquid mercury target depletes virtually all of the negatively charged pions through nuclear capture (only $\sim 2.3 \times 10^{-5}$ decay prior to nuclear capture), while the positively charged pions are rapidly slowed but not captured [44]. These particles then decay at rest, via $\pi^{+} \rightarrow \mu^{+} \nu_{\mu} \rightarrow e^{+} \nu_{e} \bar{\nu}_{\mu} \nu_{\mu}$, yielding the following isotropic spectrum per $\pi^{+}$:

$$
\begin{array}{ll}
\frac{d N_{\nu_{\mu}}}{d E_{\nu_{\mu}}}=\delta\left(E_{\nu_{\mu}}-\left(\frac{m_{\pi}^{2}-m_{\mu}^{2}}{2 m_{\pi}}\right)\right), & \\
\frac{d N_{\bar{\nu}_{\mu}}}{d E_{\bar{\nu}_{\mu}}}=\frac{6 E_{\bar{\nu}_{\mu}}^{2}}{\left(m_{\mu} / 2\right)^{4}}\left(\frac{m_{\mu}}{2}-\frac{2}{3} E_{\bar{\nu}_{\mu}}\right), & E_{\bar{\nu}_{\mu}} \leq m_{\mu} / 2, \\
\frac{d N_{\nu_{e}}}{d E_{\nu_{e}}}=\frac{12 E_{\nu_{e}}^{2}}{\left(m_{\mu} / 2\right)^{4}}\left(\frac{m_{\mu}}{2}-E_{\nu_{e}}\right), & E_{\nu_{e}} \leq m_{\mu} / 2,
\end{array}
$$

where $m_{\mu}$ and $m_{\pi}$ are the masses of the muon and pion, respectively.

After traveling to the location of the detector, these neutrinos can be observed through coherent elastic neutrinonucleus scattering. The cross section for this process is given as follows:

$$
\begin{aligned}
\frac{d \sigma}{d E_{T}}= & \frac{G_{F}^{2} M}{4 \pi}\left[N-Z\left(1-4 \sin ^{2} \theta_{\mathrm{W}}\right)\right]^{2} \\
& \times\left(1-\frac{E_{T}}{E_{\nu}}-\frac{M E_{T}}{2 E_{\nu}^{2}}+\frac{E_{T}^{2}}{2 E_{\nu}^{2}}\right) F^{2}\left(E_{T}\right),
\end{aligned}
$$

where $E_{T}$ is the recoil energy, $E_{\nu}$ is the neutrino energy, $\theta_{\mathrm{W}}$ is the weak mixing angle, $M$ is the target nuclear mass, $N(Z)$ is the number of neutrons (protons) in the nucleus, and $F$ is the Helm nuclear form factor given by the following [46]:

$$
\begin{array}{r}
F(Q)=\frac{3 j_{1}\left(R_{0} Q\right)}{R_{0} Q} \exp \left(-\frac{Q s}{2}\right), \\
j_{1}(x)=\frac{\sin x}{x^{2}}-\frac{\cos x}{x},
\end{array}
$$

where $j_{1}$ is the first-order spherical-Bessel function, $Q^{2}=-p_{\mu} p^{\mu}=2 M E_{T}$ is the momentum transfer, and $R_{0}^{2}=\sqrt{R^{2}-5 s^{2}}$ is determined by the surface thickness, $s=0.5(\mathrm{fm})$, and the effective nuclear radius, $R=$ $1.2 A^{\frac{1}{3}}(\mathrm{fm})$ [47]. In this study, we will focus on the case of CsI as our target for coherent scattering. We have neglected spin-dependent contributions to the cross section which come from axial couplings since, relative to the vector contributions, axial contributions should be suppressed by a factor of 1/A [48].

The total coherent scattering cross section is given by integrating Eq. (2) up to a maximum recoil energy of $E_{T \max }=\left(2 E_{\nu}^{2} /\left(M+2 E_{\nu}\right)\right)$. We also include a signal acceptance function, which we take to interpolate linearly between zero at $E_{T}=2.5 \mathrm{keV}$ and $100 \%$ at $E_{T}=5 \mathrm{keV}$. This represents an improvement of a factor of 2 in the midpoint $E_{T}$ over that described in Ref. [34].

By utilizing timing information, it is possible to distinguish the $\nu_{\mu}$ flux from the combined flux of $\nu_{e}$ and $\bar{\nu}_{\mu}$. In particular, the muon lifetime $\left(\tau_{\mu}=2.197 \mu \mathrm{s}\right)$ delays the $\nu_{e}$ and $\bar{\nu}_{\mu}$ arrival times to a degree that is long compared to both the pion lifetime $(0.026 \mu \mathrm{s})$ and the Gaussian pulse width $(0.16 \mu \mathrm{s})$, but short compared to the time between pulses at the Spallation Neutron Source (0.017 s). This is illustrated in Fig. 1, where we show the time profile of the neutrino luminosity from such a pulsed spallation source. These profiles are calculated by convolving the decay profile of each progenitor particle with the time profile of the beam pulse.

\section{PROJECTED SENSITIVITY TO STERILE NEUTRINOS}

The probability of a neutrino of flavor $\alpha$ oscillating to flavor $\beta$ is given by the following:

$$
P_{\alpha \rightarrow \beta}=\sum_{j, k} U_{\alpha, j}^{*} U_{\beta, j} U_{\alpha, k} U_{\beta, k}^{*} \exp \left(-i \frac{\Delta m_{j k}^{2} L}{2 E_{\nu}}\right),
$$




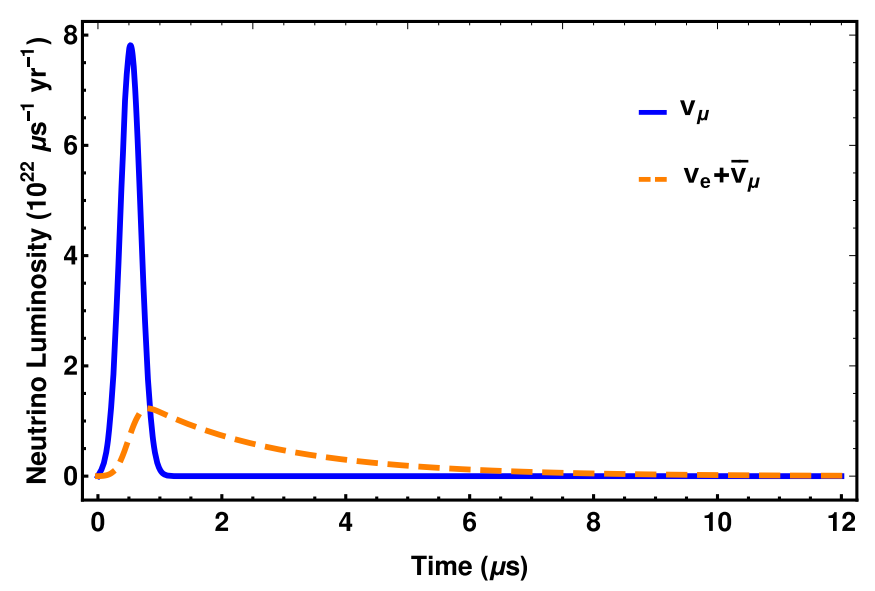

FIG. 1. The neutrino luminosity from a pulsed source of charged pions decaying at rest. The muon lifetime $\left(\tau_{\mu}=2.197 \mu \mathrm{s}\right)$ delays the $\nu_{e}$ and $\bar{\nu}_{\mu}$ emission to a degree that is long compared to the pion lifetime $(0.026 \mu \mathrm{s})$ and pulse width $(0.16 \mu \mathrm{s})$, but is very short compared to the time between pulses at the Spallation Neutron Source $(0.017 \mathrm{~s})$. This makes it possible to use timing information to separate the $\nu_{\mu}$ flux from the combined flux of $\nu_{e}$ and $\bar{\nu}_{\mu}$. Note that we have defined the time axis such that $t=0.5 \mu \mathrm{s}$ is at the center of the pulse.

where $L$ is the distance traveled, $U$ is the neutrino mixing matrix, $\Delta m_{j k}^{2}=m_{j}^{2}-m_{k}^{2}$ is the mass squared difference between two neutrino mass eigenstates, and $E_{\nu}$ is the neutrino energy. Given that we are interested in the case in which the three Standard Model neutrinos are each much lighter than the sterile state $\left(m_{1,2,3} \ll m_{4}\right)$, we can simplify the problem by setting $\Delta m_{41}^{2} \approx \Delta m_{42}^{2} \approx \Delta m_{43}^{2}$. Furthermore, Eq. (4) simplifies considerably on short baselines, for which oscillations between Standard Model (nonsterile) neutrinos are negligible. In this regime, the probability of a neutrino of flavor $\alpha$ arriving at the detector as an active neutrino is given as follows:

$$
P_{\alpha \rightarrow e, \mu, \tau}=1-4\left|U_{\alpha 4}\right|^{2}\left(1-\sum_{\beta=e, \mu, \tau}\left|U_{\beta 4}\right|^{2}\right) \sin ^{2}\left(\frac{\Delta m^{2} L}{4 E}\right),
$$

where $\Delta m^{2} \equiv \Delta m_{41}^{2} \approx \Delta m_{42}^{2} \approx \Delta m_{43}^{2}$. After appropriate unit conversions, the argument of the sine function can be rewritten as $1.27 \times\left(\Delta m^{2} / \mathrm{eV}^{2}\right)(L / \mathrm{m})(\mathrm{MeV} / E)$.

To estimate the sensitivity of a coherent scattering experiment to a sterile neutrino, we calculate the number of events predicted to be observed in four time bins, corresponding to $0-0.5,0.5-1,1-2$, and $2-10 \mu \mathrm{s}$, defined such that $t=0.5 \mu \mathrm{s}$ is at the center of the pion pulse. By taking into account this timing information, we are able to measure independently the flux of neutrinos that originate as $\nu_{\mu}$, as well and those that originate as either $\nu_{e}$ or $\bar{\nu}_{\mu}$. We consider measurements made over two baselines (20 and 40 meters), using a target consisting of $100 \mathrm{~kg}$ of CsI, over a total observation time of either 3 or 10 years (half of the
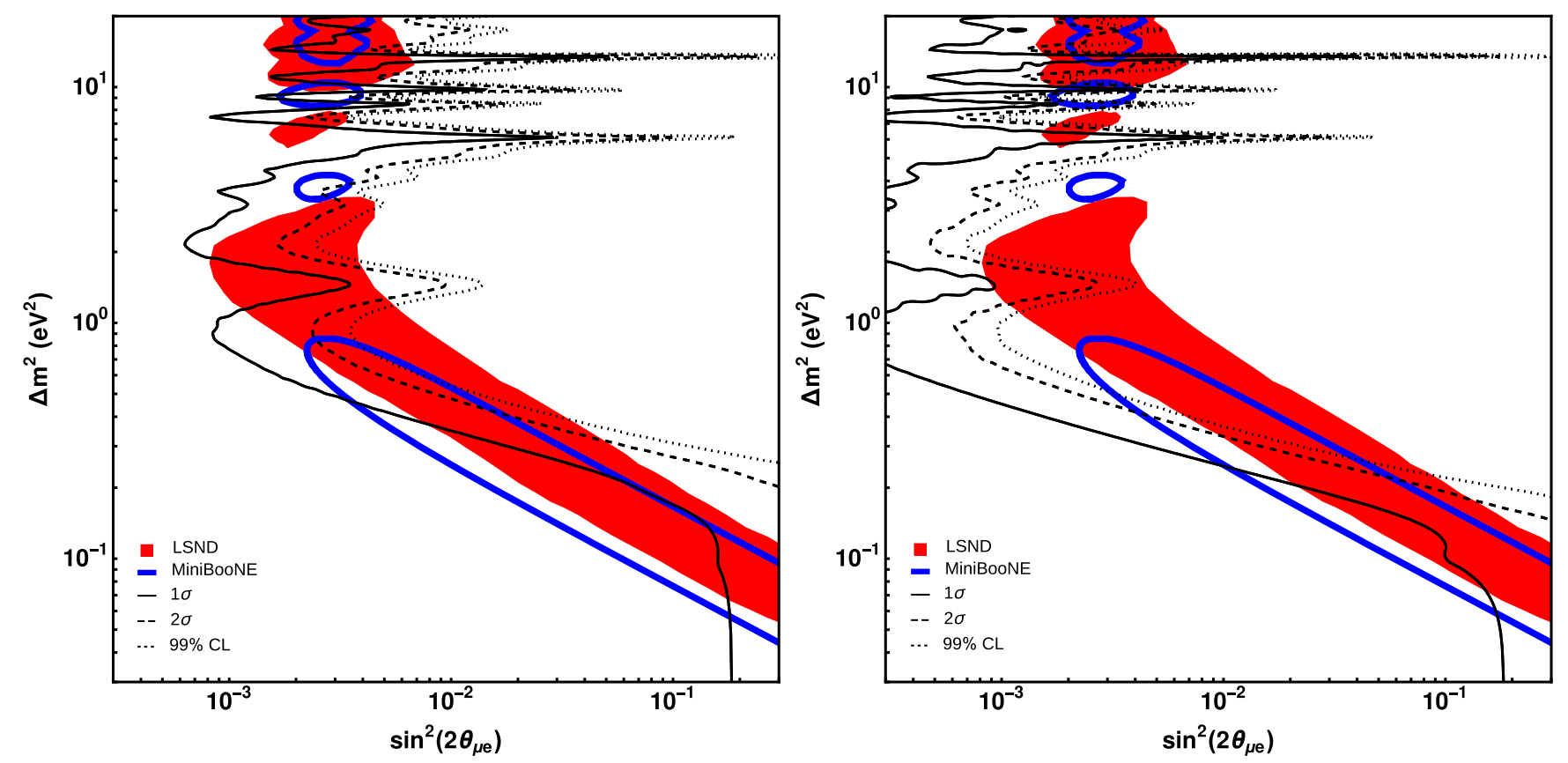

FIG. 2. The projected constraints on the sterile neutrino parameter space for a $100 \mathrm{~kg}$ CsI detector and source that generates $4 \times 10^{23}$ protons on target per year with an energy of $1 \mathrm{GeV}$, after collecting data for a total of 3 years (left) or 10 years (right). In each case, we have assumed that the detector was located at a distance of 20 meters from the source during the first half of the exposure, and at a distance of 40 meters during the second half. These constraints are compared to the regions that could potentially account for the LSND [1] and MiniBooNE [2] anomalies (at the 99\% confidence level). 
total time is assumed to be in each of the 20 and 40 meter configurations). In each configuration, we include in the event rate calculation a steady-state background of $1.45 \times 10^{-10}$ counts $/ \mathrm{kg} / \mu \mathrm{s}$, which can be precisely determined by measuring the off-pulse event rate [34]. Although this is a factor of 10 lower than the rate reported in Ref. [34], this degree of improvement is achievable through the application of additional shielding and well-understood techniques to reduce the dominant internal radiocontaminations of CsI [49] (see also Refs. [50-52]). We also include in our analysis an overall systematic uncertainty of $\pm 28 \%$ on the overall signal rate, corresponding to uncertainties associated with the cross section, detector efficiency, and overall neutrino flux. This uncertainty budget is adopted from the experimental analysis performed by the COHERENT collaboration using a detector technology equivalent to the one described herein. [34] We define a $\chi^{2}$ as follows:

$\chi^{2}=\sum_{t} \frac{\left(N_{t}^{0}-N_{t}\left(U_{e 4}, U_{e 4}, \Delta m^{2}\right)(1+\alpha)\right)^{2}}{N_{t}^{0}+2 B_{s s}}+\left(\frac{\alpha}{0.28}\right)^{2}$
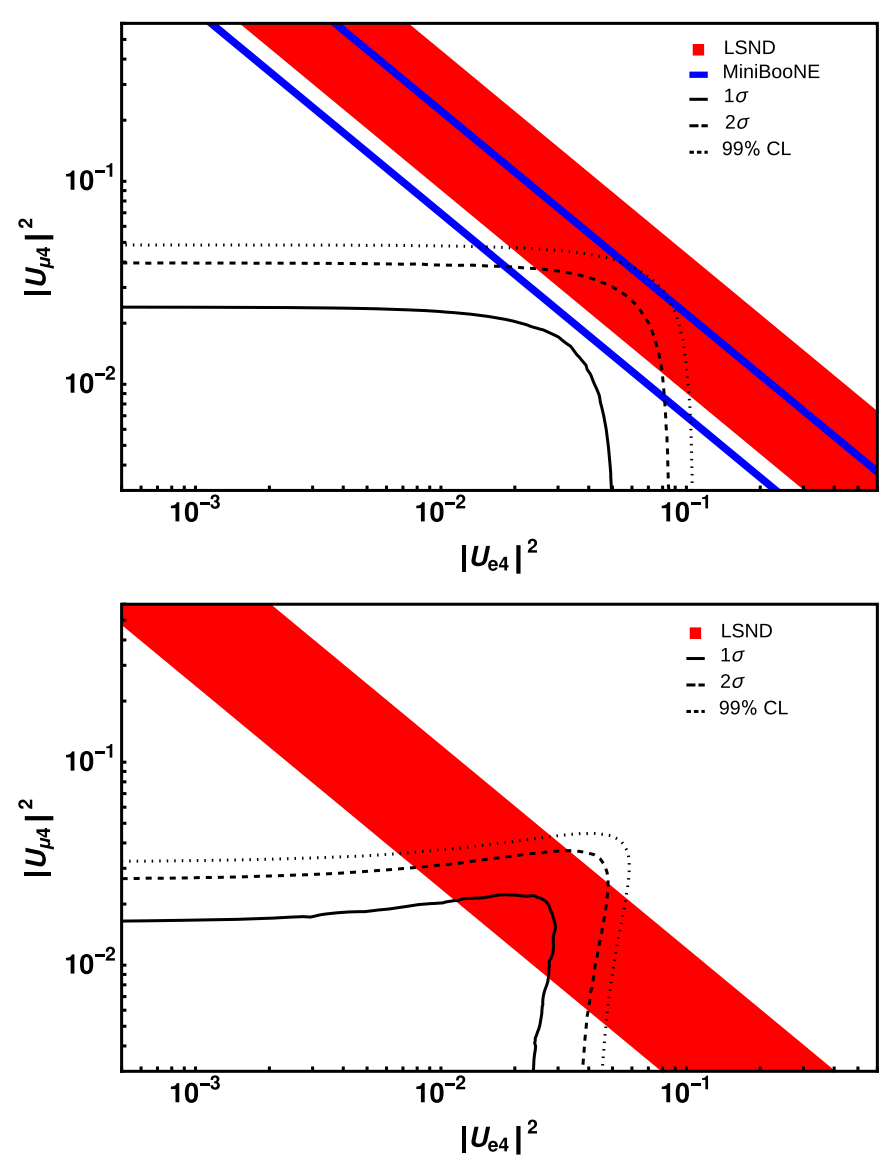

where the sum is taken over the time bin described above, $N^{0}$ is the expected number of events without sterilemediated oscillations, $N_{t}\left(U_{e 4}, U_{e 4}, \Delta m^{2}\right)$ is the number of events expected as a function of the sterile mixing matrix elements and mass splitting, $\alpha$ is a systematic parameter corresponding to the uncertainty in the signal rate, and $B_{s s}$ is the estimated steady-state background. We minimize the $\chi^{2}$ over $\alpha$ for values of $U_{e 4}, U_{e 4}, \Delta m^{2}$ during our analysis in order to obtain our sensitivity contours.

Additionally, the systematic uncertainties associated with the flux normalization, coherent scattering cross section, and detector efficiencies could be reduced significantly by taking ratios of the event rates at the two baselines and calibrating the detectors in order to understand any possible differences in their response functions [43].

The main results of our analysis are shown in Figs. 2 and 3. In the first of these figures, we show the projected constraints on the sterile neutrino parameter space from an experiment utilizing a $100 \mathrm{~kg}$ CsI detector and source producing a luminosity of $4 \times 10^{23}$ protons on target per year with an energy of $1 \mathrm{GeV}$. We present this result in terms of the effective mixing parameter $\sin ^{2}\left(2 \theta_{\mu e}\right) \equiv 4\left|U_{e 4}\right|^{2}\left|U_{\mu 4}\right|^{2}$. For simplicity, we have limited
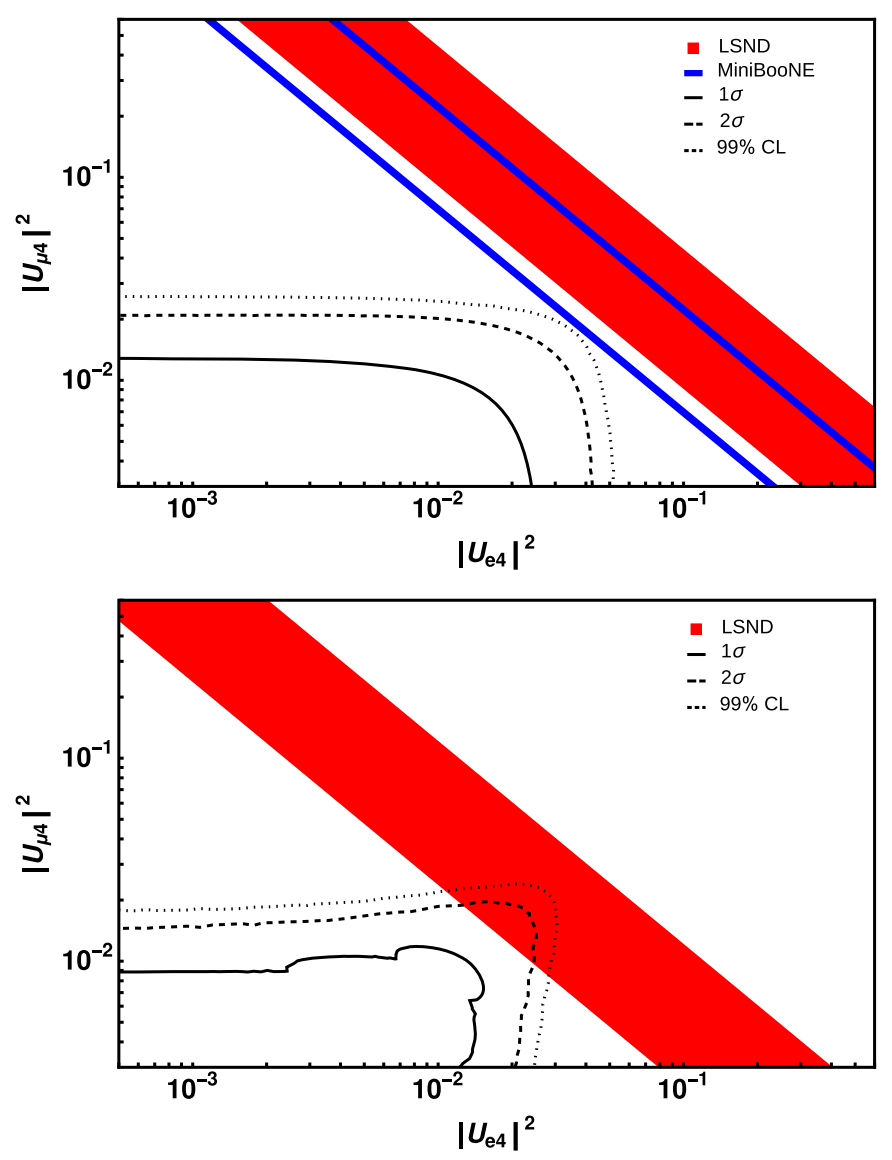

FIG. 3. As in Fig. 2, but in terms of the $\left|U_{\mu 4}\right|^{2}$ vs $\left|U_{e 4}\right|^{2}$ parameter space, for the choice of either $\Delta m^{2}=0.55 \mathrm{eV}^{2}$ (upper frames) or $\Delta m^{2}=1.3 \mathrm{eV}^{2}$ (lower frames). Again, the left (right) frames are after collecting data for 3 (10) years. These constraints are compared to the regions that could potentially account for the LSND [1] and MiniBooNE [2] anomalies (at the 99\% confidence level). 
our discussion to the case of $\left|U_{\tau 4}\right|=0$. In Fig. 3, we show these constraints in the $\left|U_{\mu 4}\right|^{2}$ vs $\left|U_{e 4}\right|^{2}$ plane, for two choices of $\Delta m^{2}$. When these constraints are compared to the regions favored by LSND and MiniBooNE, we conclude that the search proposed here would be sensitive to the vast majority of the sterile neutrino parameter space that could potentially account for these anomalies. Note that timing information gives this analysis sensitivity to $\left|U_{\mu 4}\right|^{2}$ and $\left|U_{e 4}\right|^{2}$ approximately independently. Since this can disentangle the mixing of a sterile neutrino with electron and muon neutrinos, the sensitivity to oscillation will carve out approximately vertical and horizontal regions in the $\left|U_{\mu 4}\right|^{2}$ vs $\left|U_{e 4}\right|^{2}$ plane which is particularly effective in probing the otherwise diagonal best-fit region.

In these projections, we have considered measurements taken over baselines of 20 and 40 meters. If the separation between these distances were increased (decreased), the exclusion contours would shift downward (upward) in $\Delta m^{2}$, as a consequence of the dependence on $L$ and $\Delta m^{2}$ in Eq. (5).

\section{SUMMARY AND CONCLUSIONS}

In this paper, we have proposed using a $100 \mathrm{~kg} \mathrm{CsI}$ coherent neutrino detector located near a pulsed source of neutrinos functionally similar to the Spallation Neutron Source at the Oak Ridge National Laboratory to test sterile neutrino scenarios motivated by the LSND and MiniBooNE anomalies. By making use of timing information, one independently measures the fluxes of neutrinos that originate as $\nu_{\mu}$ or as either $\nu_{e}$ or $\bar{\nu}_{\mu}$. Furthermore, by comparing the coherent scattering rates observed by a given detector while positioned at multiple distances from the source, it is possible to significantly reduce systematic uncertainties associated with the flux normalization, coherent scattering cross section, and detector efficiencies. Alternatively, two $50 \mathrm{~kg}$ CsI detectors could be used to simultaneously take data and therefore reduce any time dependent flux uncertainty. We find that such an experiment would be sensitive to much of the relevant parameter space and would help to clarify the nature of the mysterious results reported by the LSND and MiniBooNE Collaborations.

\section{ACKNOWLEDGMENTS}

We would like to thank Juan Collar for helpful discussions. C. B. is supported by the U.S. National Science Foundation Graduate Research Fellowship under Grants No. DGE-1144082 and No. DGE-1746045. This manuscript has been authored by Fermi Research Alliance, LLC, under Contract No. DE-AC02-07CH11359 with the U.S. Department of Energy, Office of Science, Office of High Energy Physics.
[1] A. Aguilar-Arevalo et al. (LSND Collaboration), Phys. Rev. D 64, 112007 (2001).

[2] A. A. Aguilar-Arevalo et al. (MiniBooNE Collaboration), Phys. Rev. Lett. 121, 221801 (2018).

[3] M. Dentler, Á. Hernndez-Cabezudo, J. Kopp, P. A. N. Machado, M. Maltoni, I. Martinez-Soler, and T. Schwetz, J. High Energy Phys. 08 (2018) 010.

[4] J. Kopp, M. Maltoni, and T. Schwetz, Phys. Rev. Lett. 107, 091801 (2011).

[5] J. Kopp, P. A. N. Machado, M. Maltoni, and T. Schwetz, J. High Energy Phys. 05 (2013) 050.

[6] M. Dentler, Á. Hernndez-Cabezudo, J. Kopp, M. Maltoni, and T. Schwetz, J. High Energy Phys. 11 (2017) 099.

[7] J. N. Bahcall, Phys. Rev. C 56, 3391 (1997).

[8] M. A. Acero, C. Giunti, and M. Laveder, Phys. Rev. D 78, 073009 (2008).

[9] C. Giunti and M. Laveder, Phys. Rev. C 83, 065504 (2011).

[10] W. Hampel et al. (GALLEX Collaboration), Phys. Lett. B 447, 127 (1999).

[11] J. N. Abdurashitov et al., Astropart. Phys. 25, 349 (2006).

[12] G. Mention, M. Fechner, T. Lasserre, T. A. Mueller, D. Lhuillier, M. Cribier, and A. Letourneau, Phys. Rev. D 83, 073006 (2011).
[13] P. Huber, Phys. Rev. C 84, 024617 (2011); 85, 029901(E) (2012).

[14] M. G. Aartsen et al. (IceCube Collaboration), Phys. Rev. Lett. 117, 071801 (2016).

[15] P. Adamson et al. (MINOS Collaboration), Phys. Rev. Lett. 122, 091803 (2019).

[16] G. H. Collin, C. A. Argelles, J. M. Conrad, and M. H. Shaevitz, Nucl. Phys. B908, 354 (2016).

[17] S. Gariazzo, C. Giunti, M. Laveder, and Y. F. Li, J. High Energy Phys. 06 (2017) 135.

[18] E. Bertuzzo, S. Jana, P. A. N. Machado, and R. Z. Funchal, Phys. Rev. Lett. 121, 241801 (2018).

[19] E. Bertuzzo, S. Jana, P. A. N. Machado, and R. Z. Funchal, Phys. Lett. B 791, 210 (2019).

[20] P. Ballett, S. Pascoli, and M. Ross-Lonergan, Phys. Rev. D 99, 071701 (2019).

[21] C. A. Argelles, M. Hostert, and Y.-D. Tsai, Phys. Rev. Lett. 123, 261801 (2019).

[22] A. A. Aguilar-Arevalo et al. (MiniBooNE DM Collaboration), Phys. Rev. D 98, 112004 (2018).

[23] J. R. Jordan, Y. Kahn, G. Krnjaic, M. Moschella, and J. Spitz, Phys. Rev. Lett. 122, 081801 (2019).

[24] N. Arkani-Hamed, S. Dimopoulos, G. R. Dvali, and J. March-Russell, Phys. Rev. D 65, 024032 (2001). 
[25] N. Arkani-Hamed and M. Schmaltz, Phys. Rev. D 61, 033005 (2000).

[26] A. Lukas, P. Ramond, A. Romanino, and G. G. Ross, Phys. Lett. B 495, 136 (2000).

[27] R. Barbieri, P. Creminelli, and A. Strumia, Nucl. Phys. B585, 28 (2000).

[28] H. Davoudiasl, P. Langacker, and M. Perelstein, Phys. Rev. D 65, 105015 (2002).

[29] P. A. N. Machado, H. Nunokawa, and R. Z. Funchal, Phys. Rev. D 84, 013003 (2011).

[30] P. A. N. Machado, H. Nunokawa, F. A. P. dos Santos, and R. Z. Funchal, Phys. Rev. D 85, 073012 (2012).

[31] A. Esmaili and O. L. G. Peres, Phys. Rev. D 85, 117301 (2012).

[32] M. Carena, Y.-Y. Li, C. S. Machado, P. A. N. Machado, and C. E. M. Wagner, Phys. Rev. D 96, 095014 (2017).

[33] J. E. Hill, Phys. Rev. Lett. 75, 2654 (1995).

[34] D. Akimov et al. (COHERENT Collaboration), Science 357, 1123 (2017).

[35] J. Collar, N. Fields, M. Hai, T. Hossbach, J. Orrell, C. Overman, G. Perumpilly, and B. Scholz, Nucl. Instrum. Methods Phys. Res., Sect. A 773, 56 (2015).

[36] A. J. Anderson, J. M. Conrad, E. Figueroa-Feliciano, C. Ignarra, G. Karagiorgi, K. Scholberg, M. H. Shaevitz, and J. Spitz, Phys. Rev. D 86, 013004 (2012).

[37] T. S. Kosmas, D. K. Papoulias, M. Tortola, and J. W. F. Valle, Phys. Rev. D 96, 063013 (2017).
[38] B. Dutta, Y. Gao, R. Mahapatra, N. Mirabolfathi, L. E. Strigari, and J. W. Walker, Phys. Rev. D 94, 093002 (2016).

[39] B. C. Caas, E. A. Garcs, O. G. Miranda, and A. Parada, Phys. Lett. B 776, 451 (2018).

[40] J. A. Formaggio, E. Figueroa-Feliciano, and A. J. Anderson, Phys. Rev. D 85, 013009 (2012).

[41] M. Elnimr et al. (OscSNS Collaboration), The OscSNS white paper, arXiv:1307.7097.

[42] F. T. Avignone and Y. V. Efremenko, J. Phys. G 29, 2665 (2003).

[43] L. A. Mikaélyan and V. V. Sinev, Phys. At. Nucl. 63, 1002 (2000).

[44] F. T. Avignone and Y. V. Efremenko, J. Phys. G 29, 2615 (2003).

[45] D. Y. Akimov et al., Phys. Procedia 74, 411 (2015).

[46] J. D. Lewin and P. F. Smith, Astropart. Phys. 6, 87 (1996).

[47] D. K. Papoulias and T. S. Kosmas, Phys. Rev. D 97, 033003 (2018).

[48] J. Barranco, O. G. Miranda, and T. I. Rashba, J. High Energy Phys. 12 (2005) 021.

[49] Juan Collar (private communication).

[50] H. J. Kim et al., IEEE Trans. Nucl. Sci. 55, 1420 (2008).

[51] T. Y. Kim et al., Nucl. Instrum. Methods Phys. Res., Sect. A 500, 337 (2003).

[52] Y. D. Kim et al., Nucl. Instrum. Methods Phys. Res., Sect. A 552, 456 (2005). 